\title{
Fine-Structural Alterations and Clustering of Developing Synapses after Chronic Treatments with Low Levels of NMDA
}

\author{
Lai-Hsing Yen, Jane T. Sibley, and Martha Constantine-Paton \\ Department of Biology, Yale University, New Haven, Connecticut 06511
}

In the visual pathway of frogs it is possible to apply low levels of NMDA chronically to the optic tectum and study the mechanisms underlying the stabilization of synapses developing within the CNS. Earlier studies (Cline and Constantine-Paton, 1990) found that chronic NMDA treatment of tecta innervated by two retinas results in a reduction of branching within the terminal arbors of retinal ganglion cells (RGCs). We now report that this same chronic NMDA treatment produces fine-structural changes in synaptic morphology as well as local synaptic rearrangements within the retinotectal neuropil.

Chronic NMDA treatment of doubly innervated tecta was associated with a thickening or darkening of both pre- and postsynaptic densities. These changes in synapse morphology were restricted to the superficial neuropil of tecta in regions where reductions in branches of RGC axonal arbors were observed at the light microscopic level. The finestructural effects were absent from similarly treated tecta innervated by only one eye, where RGC axonal arbor pruning was not observed. Stereological analyses indicated that the incidence of two or more presynaptic profiles converging on the same postsynaptic process was significantly increased in the NMDA-treated, doubly innervated tecta. This observed increase in synaptic clustering was not associated with a larger synaptic active zone, or with an increase in the number of synapses per unit volume.

These data are discussed in the context of the hypothesis that chronic NMDA treatment raises the threshold for synapse stabilization in tectal neurons, causing the selective loss of poorly correlated synapses of both retinal and nonretinal origin from tectal neuropil that is innervated by two retinas: increased pre- and postsynaptic thickening could reflect greater efficiency in the remaining synaptic contacts and their closer spatial proximity on the same postsynaptic process is consistent with greater cooperativity and less competition.

IKey words: NMDA receptor, retinotectal projection, correlated activity, three-eyed frogs, synaptic morphology, synaptic clustering, quantitative electron microscopy]

\footnotetext{
Received July 28, 1992; revised May 10, 1993; accepted May 24, 1993.

We thank Dr. J. Norden and Dr. S. Hockfield for critical reviews of the manuscript, and we are grateful to Dr. M. Mooseker for allowing us to use his FM facility and to Dr. K.-Y. Wei for allowing use of his morphometric measurement system. This work was supported by NIH Grant EY08760.

Correspondence should be addressed to Dr. Martha Constantine-Paton, Department of Biology, P.O. Box 6666, Yale University, New Haven, CT 06511.

Copyright (C) 1993 Society for Neuroscience $0270-6474 / 93 / 134949-12 \$ 05.00 / 0$
}

A variant of the Hebbian rule for cellular learning has frequently been invoked as an explanation for activity-dependent synaptic competition and synapse elimination during development. According to this postulate, afferents that can cooperate to depolarize their postsynaptic partners significantly are reinforced or stabilized such that they remain into adulthood, while inputs unable to evoke such a depolarization are destabilized and lost (Stent, 1973; Changeaux and Danchin, 1976). Considerable data from developing sensory systems are consistent with this idea. Most recently, the results of experiments in which regions of the developing CNS are chronically treated with 5-aminophosphonovaleric acid (AP5), the specific antagonist of the NMDA subtype of glutamate receptor, suggest that the normal function of this receptor is critical to the synapse stabilization process (Cline et al., 1987; Kleinschmidt et al., 1987; Bear et al., 1990; Hahm et al., 1991). According to this interpretation, one important parameter in determining which young and otherwise transient synapses survive to participate in maturing circuitry is the amount of target cell NMDA receptor activation they induce.

In developing frogs it is possible to apply low levels of NMDA chronically to the optic tectum and change both the distribution and morphology of retinal ganglion cell (RGC) terminal arbors (Cline and Constantine-Paton, 1990). These alterations are observed only in tectal lobes doubly innervated by a host and a supernumerary retina, where previous studics (Rch and Constantine-Paton, 1985; Constantine-Paton and Ferrari-Eastman, 1987; J. J. Norden and M. Constantine-Paton, unpublished observations) have indicated that the overall degree of correlated activity among converging inputs should be lower than in tecta innervated by one eye. Within these NMDA-treated doubly innervated tecta, the synapse-bearing branches of RGC arbors are pruned at stripe borders where uncorrelated inputs from two eyes mix (Cline and Constantine-Paton, 1990). Electrophysiological studies on similarly treated tecta indicate that the functional effectiveness of the NMDA receptor system has been reduced (Debski et al., 1991; Hickmott and Constantine-Paton, 1991). These observations are consistent with the hypothesis that activation of the NMDA receptor is a critical trigger in the cascade of events that stabilizes converging synapses on the basis of their correlated activity: decreased NMDA receptor effectiveness would be expected to remove developing synapses from regions of low correlated activity, because under those conditions the amount of correlation among converging afferents necessary to produce a sustaining level of current through the NMDA receptor channel should increase. Thus, a larger proportion of moderately correlated converging inputs would fall below stabilization threshold and be lost or "pruned." 
This corollary of the NMDA receptor hypothesis for stabilizing developing synapses has important implications. For example, it could provide a mechanism for reducing the synaptic rearrangements and thus links the regulation of this receptor system to the control of structural plasticity during development. In addition, refinement of projections during normal development would be expected to increase NMDA receptor activation because refinement is likely to be associated with increased activity correlations among neighboring afferents. Increases in the cooperative activation of NMDA receptors might be expected to initiate their downregulation by postsynaptic neurons. As downregulation occurs, inputs that had not collected highly cooperative neighbors would be eliminated and new inputs that do not, by chance, establish synapses adjacent to existing contacts with which they are highly correlated will be withdrawn.

The potential developmental significance of NMDA receptor downregulation and the possibility that the frog could provide a system where this has been brought under experimental control to produce a structural change in synaptic circuitry prompted us to undertake electron microscopic (EM) analyses of NMDA-treated frog tecta. According to the hypothesis for synapse stabilization described above, the synaptic active sites that remain in these tecta should be effective at driving their postsynaptic partners and they should also have high activity correlations with converging neighbors. Here we report two pronounced changes in the fine structure of the NMDA-treated, doubly innervated tecta: the pre- and the postsynaptic densities are darker or thicker at individual active zones, and the incidence with which two or more active zones converge on the same postsynaptic process is significantly increased. These alterations are consistent with the postulate that the synapses that survive in these neuropils are very effective release sites and that converging inputs are more cooperative than their counterparts in unaffected tecta.

\section{Materials and Methods}

Animals. The animals used in these studies were offspring of adult wildcaught Rana pipiens purchased from Hazen \& Sons, Alburg, VT. Embryos were obtained by induced ovulation and artificial fertilization in the laboratory using standard techniques. The embryonic microsurgery used to produce doubly innervated tecta has been detailed in a previous publication (Law and Constantine-Paton, 1981). Briefly, one eye primordium of an early to midstage embryo (Shumway stages 16-19) was transferred to the evacuated position of the olfactory placode in a similarly staged sibling host. The host embryo was pushed into a depression in the wax bottom of an operating dish containing $100 \%$ Steinberg's solution for approximately $15 \mathrm{~min}$ until the graft healed in place. Tadpoles were raised in deionized water containing $0.05 \%$ instant ocean salt and fed boiled romaine lettuce. When the front legs emerged the metamorphosing animals were placed in a shallow tilted container to prevent drowning. Postmetamorphic frogs were fed liberally on Drosophila melanogaster.

Chronic treatment. NMDA in concentrations that have previously been shown to prune developing retinal ganglion cell arbors without producing degenerative damage to the arbors or loss of retinal or tectal neurons (Cline and Constantine-Paton, 1990) was applied to tecta of Taylor and Kollros (1946) stage XXIV-XXV froglets. The infiltration of Elvax slabs (a gift of Du Pont) with NMDA was accomplished by adding $10 \mu \mathrm{l}$ of $10^{-2} \mathrm{M}$ NMDA solution in distilled water along with $10 \mu \mathrm{l}$ of $2 \%$ fast green in dimethyl sulfoxide to $1 \mathrm{ml}$ of the Elvax solution ( $100 \mathrm{mg}$ of Elvax dissolved in $1 \mathrm{ml}$ of methylene chloride). The solution was then frozen and cold-vacuumed to remove the organic solvent (Silberstein and Daniel, 1982). Thin slices of Elvax approximately 1000 $\times 1000 \times 60 \mu \mathrm{m}$ were cut on a cryostat. Based on previous measurements (Cline and Constantine-Paton, 1989) we have calculated that these Elmax slices implanted on each animal should release molecules in the molecular weight range of NMDA at a rate of approximately $0.005 \mathrm{nmol} / \mathrm{d}$.

Animals were anesthetized for surgery by submersion in $0.05 \%$ 3-aminobenzoic acid ethyl ester (MS-222; Sigma). The skin, cartilaginous skull, dura, and arachnoid membranes overlying the dorsal surface of the tectal lobes were retracted and the Elvax slices containing NMDA were positioned to cover completely the dorsal and lateral surfaces of both tectal lobes. The dura and cartilage were then replaced and the skin was sealed with Histoacryl glue (Tri-Hawk, Montreal, Quebec, Canada). Animals were allowed to survive the treatment for 4-5 weeks. For sham-treated control animals, Elvax lacking NMDA but otherwise prepared and applied in the same way was uscd. Threc NMDA-treated and three sham-treated doubly innervated tectal lobes in animals that survived all treatments and had successful anterograde labeling were examined in this study. Additionally, two normal two-eyed postmetamorphic frogs at the same developmental stage were also treated with NMDA in exactly the same way for comparison of synaptic structure.

Axon terminal reconstruction. In a previous study employing chronic NMDA treatment, we encountered one group of animals all treated chronically with the same batch of NMDA-infiltrated Elvax that did not show structural effects on RGC terminals in their doubly innervated tecta. The efficacy of NMDA synaptic transmission in animals from this same group was analyzed physiologically and found to also lack the decrease in efficacy otherwise always observed in NMDA-treated tecta. We therefore concluded that the NMDA in that batch of Elvax had lost its potency probably because it had been stored for an unusually long period before use (Debski et al., 1991). This incident alerted us to the need to control the present analyses involving chronic NMDA treatment for the possible loss of NMDA potency. Consequently, to ensure that all analyses were conducted in doubly innervated regions of all tecta, as well as to verify that the released NMDA in each case had the effect described previously at the level of the light microscope (Cline and Constantine-Paton, 1990), we labeled a few retinal ganglion cell (RGC) terminals arising from the ventral-nasal region of the supernumerary eye of all animals by placing a pellet of the enzyme horseradish peroxidase (HRP) in that region of the retina $2 \mathrm{~d}$ before death. This was accomplished by coating the tip of a fine insect pin with a saturated solution of HRP, and inserting the pin into the supernumerary retina of an anesthetized animal. The animal was recovered in oxygen-saturated water and, $2 \mathrm{~d}$ later, it was deeply anesthetized and its tecta were subsequently removed. The tecta were fixed in $0.1 \mathrm{M}$ cacodylate buffer containing $2.5 \%$ glutaraldehyde and $1 \%$ paraformaldehyde overnight at $4^{\circ} \mathrm{C}$, and then cut into $40-\mu \mathrm{m}$-thick sections and reacted with diaminobenzidine (Sigma). Individual slices were then dehydrated, postfixed in $0.1 \mathrm{M}$ cacodylate buffer containing $1 \%$ osmium tetroxide and $1.5 \%$ potassium ferricyanide, and then embedded in Spurr's solution on glass slides coated with liquid releasing agent (EM Sciences). The slides were subsequently cured at $70^{\circ} \mathrm{C}$ overnight. The morphology of isolated HRPlabeled type IV RGC (Stirling and Merrill, 1987) axons in the caudalmedial region of striped tecta were reconstructed from several of these embedded sections using a $63 \times$ oil immersion lens and a camera lucida drawing tube.

Quantitative measurements of synapse size and density. Regions containing, or closely adjacent to, the labeled arbors were cut on a Sorvall MT-2 ultramicrotome. Ultrathin sections with gold color, which are approximately $90 \mathrm{~nm}$ thickness, were stained with uranyl acetate and lead citrate and observed under a Zeiss-10 transmission electron $\mathrm{mi}-$ croscope. Nonconsecutive ultrathin sections separated by at least 0.9 $\mu \mathrm{m}$ were placed on 400 mesh grids (EM Sciences). In each ultrathin section, roughly 10 equally separated grid squares containing the superficial neuropil were selected for photographing. EM pictures of a 9.1 $\times 7.7 \mu \mathrm{m}^{2}$ area were taken in one specific corner of each selected grid square. Since these squares fell randomly across the different laminae of this neuropil, the procedure provided a randomized collection of electron micrographs of synapses in tectal layers 7, 8, and 9, where the retinal ganglion cell projections terminate (Székely and Lázár, 1976). The entire EM analysis was limited to the doubly innervated caudalmedial region of all tecta to ensure consistency. Though all of the morphometric measurements were taken using gold ultrathin sections (approximately $90 \mathrm{~nm}$ in thickness), high-power photomicrographs were also taken using silver sections (approximately $60 \mathrm{~nm}$ in thickness) to illustrate better the fine structural details.

For measuring the mean length of synaptic active zones, individual synaptic active zone lengths were highlighted and fed into a Zenith (Data System) computer through a CCD camera (Ikegami). The length of 
synaptic active zones was then automatically measured by the morphometric software package OPTIMAS. Few perforated synaptic traces (discontinuous synaptic active zones) were encountered. When they were observed, a perforated synaptic trace was considered as a single synapse and its synaptic length was quantified as the sum of the individual synaptic trace lengths.

For measuring the synaptic density, we employed the disector method that is not biased by the size, shape, or orientation of synapses (Sterio, 1984; Gundersen et al., 1988; Coggeshall, 1992). For each neuropil examined, a long series of ultrathin sections were collected on Formvarcoated slot grids. The thickness of each section was determined by the interference colors based on measurements done by Peachey (1958). Within each series, at least six pairs of adjacent sections (a "reference" section and a "look-up" section) were chosen for detailed analysis. Each pair was separated from the next pair by at least $1.5 \mu \mathrm{m}$ to avoid having the same synapse sampled. Areas falling within layers 7-9 of the tectal neuropil on the reference ultrathin sections were systematically chosen and photographed. The same areas on the adjacent look-up sections were then photographed using detailed maps of cell bodies and blood vessels as landmarks. The tissue volumes encompassed by each area times the sum of the thickness of the reference section plus the lookup section are called disector volumes. In each chosen area (for each disector volume) on the sections, the number of synapses appearing in the reference section and not in the look-up section was counted. In the disector analysis such a profile is called a "TOP." For each disector volume this analysis was performed in both directions by interchanging the roles of reference and look-up sections. Synaptic density for a neuropil was then calculated as the sum of all TOPs divided by the sum of all disector volumes.

Synaptic clustering analyses. We define "clustering" with respect to the number of presynaptic profiles associated with the same postsynaptic profile and appearing in a single ultrathin section. Thus " 1 on 1 " refers to a single presynaptic profile on to a single postsynaptic profile, while " 2 on 1 " refers to two presynaptic profiles converging on the same postsynaptic profile, and so on. Clusters of up to five synaptic contacts on to a single postsynaptic profile were seen in some of this material. The criteria for synapse identification were an active zone constituted of apposed pre- and postsynaptic densities, and synaptic vesicles associated with the presynaptic density. We scored synapses and categorized them for synaptic "clustering" in each photograph with field area of $9.1 \mu \mathrm{m} \times 7.7 \mu \mathrm{m}$ as described above. A minimum neuropil area of $5835 \mu \mathrm{m}^{2}$ per animal and a maximum neuropil area of $110,570 \mu \mathrm{m}^{2}$ per animal was examined in this analysis (see Table 2 for amount of area and total number of synapses surveyed for each animal).

\section{Results}

A previous study using chronic NMDA treatment of frog tecta demonstrated that one of the most pronounced effects of the treatment at the level of single RGC terminals was a reduction in the number of the terminal branches. This reduction was quantified at the light microscope level as the number of branch endings per unit tangential area covered by the arbor (Cline and Constantine-Paton, 1990). For the present study we used the light microscope analysis of individual terminals to assure that the NMDA treatments in this and the earlier work were producing similar effects. In addition, HRP was placed only in the supernumerary retinas and the areas examined by EM were taken from the region of tecta occupied by and immediately surrounding the labeled RGC terminals. This procedure assured that the tectal regions subsequently used in the stereological EM analyses had input from the supernumerary retina. We know from many previous studies on three-eyed frogs that the contralateral host eye always fully innervates striped tecta. Thus, by confirming innervation from the supernumerary eye in the region we examined, we assured that the region of tectum was "doubly innervated." However, though the region we examined was invariably larger than the dimension of one stripe, there was no fine-structural evidence of a "stripe boundary" in this material, and we consequently have no way of knowing whether

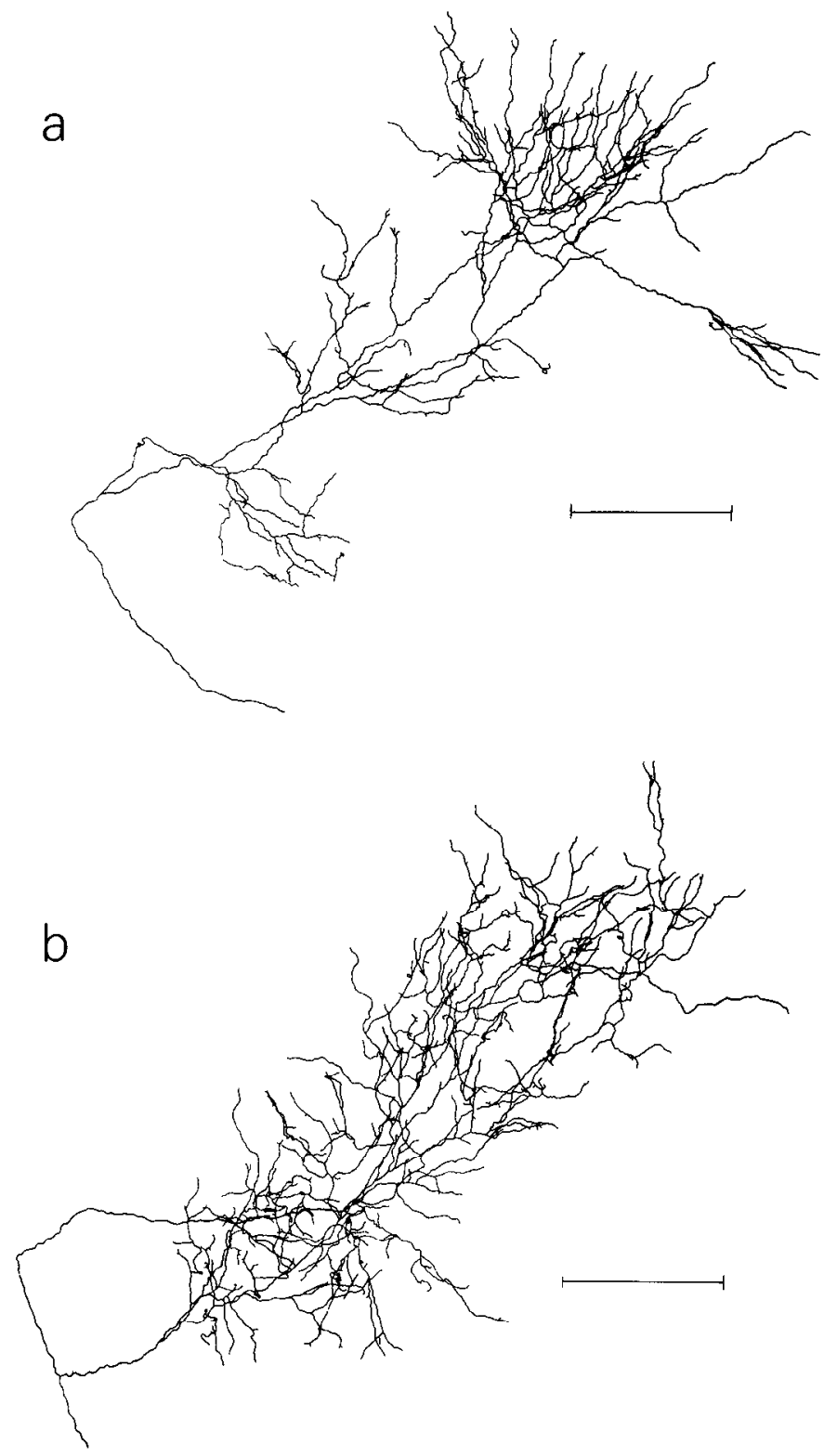

Figure 1. Light microscopic reconstructions of axonal morphologies of type IV RGCs in doubly innervated tecta. The terminals are reconstructed from serial Epon-embedded sections taken tangential to the medial-caudal surface of the tecta. They show the branch loss in NMDAtreated tecta, which we used to verify that the treatment used in this EM analysis had the effect found previously at the light microscope level. $a$, An NMDA-treated axonal terminal. $b$, A sham-treated axonal terminal. Scale bars, $100 \mu \mathrm{m}$.

any particular sample area fell in a zone where inputs from the host or the supernumerary eye predominated. This approach was favored over the alternative of placing enough HRP in the supernumerary optic nerve to label the entire projection and fully define "stripes," because the latter produced very dark HRP reaction product that obscured distinctions and accurate quantitation of synapses. Dense filling the entire projection with HRP also prevents the reconstruction of individual RGC arbor terminals to document NMDA-induced branch pruning.

Figure 1 illustrates a pair of RGC axonal terminals reconstructed from several epon embedded sections in one NMDAtreated postmetamorphic frog, and in one sham-treated control of identical age. One difference between these light microscope 

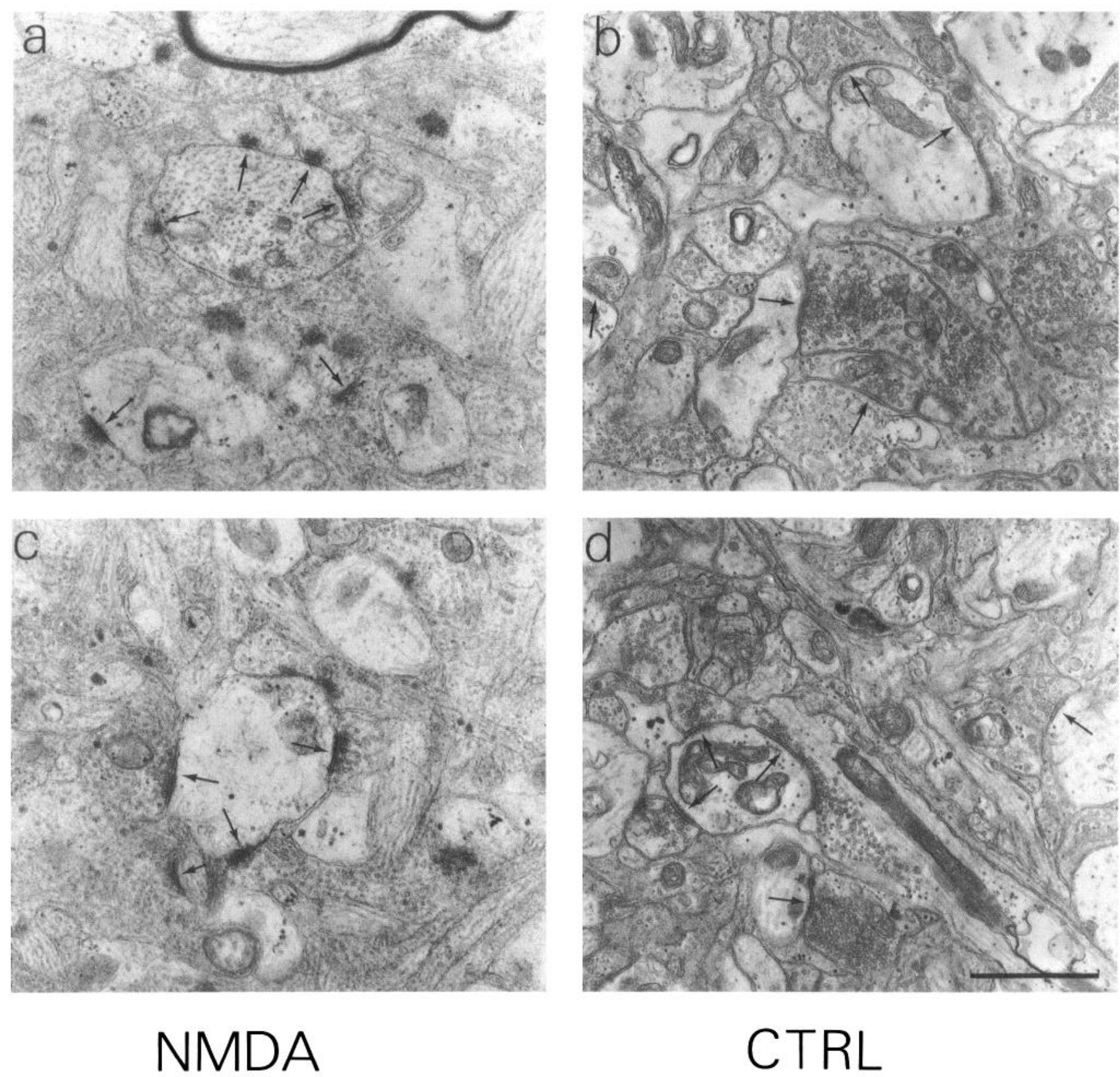

Figure 2. Synaptic morphology after NMDA treatment. $a$ and $c$ are low-power fields in layer 8 of the superficial neuropil in NMDA-treated, doubly innervated tecta. $b$ and $d$ are similar fields in sham-treated, doubly innervated tecta. Arrows point from postsynaptic to presynaptic profiles. All EM ultrathin sections photographed here were approximately $90 \mathrm{~nm}$ thick. With the same uranyl acetate and lead citrate staining, pre- and postsynaptic density appeared thicker in NMDA-treated tecta as compared to sham-treated control tecta. $b$ and $d$ are printed with more contrast and darker than $a$ and $c$ to ensure that the synaptic effects are not due to printing variation. Scale bar, $1 \mu \mathrm{m}$.

data and that of the previous work is that here we have concentrated the reconstructions only on the type IV class of RGC axonal arbors. These arbors, believed to arise from the dimming detectors of RGCs (Stirling and Merrill, 1987), project deep to layer 8 of the tectum. They are large and myelinated, and they constitute a relatively homogeneous population that can be identified from animal to animal. Quantitative comparison of these arbors indicates that there are twice as many branch endings in the control as in the NMDA-treated tissue ( 350 vs 151 branch endings), suggesting that branch loss has occurred with NMDA treatment. This is fully consistent with the results obtained in the earlier study (Cline and Constantine-Paton, 1990).
Upon inspection under the EM, the most striking aspect of NMDA-treated tecta versus sham-treated control tecta was a thickening or darkening of both pre- and postsynaptic densities in the majority of synapses. This morphological change in the active zone is shown in low-power fields of gold ultrathin sections in Figure 2 ( $a$ and $c$, NMDA treated; $b$ and $d$, sham-treated control). The thickening of the pre- and postsynaptic densities was seen in synapses showing a range of synaptic types as can be seen in high-power fields of silver ultrathin sections in Figure $3 a-f(a, c$, and $e$, NMDA treated; $b, d$, and $f$, sham-treated control). Figure 3, $a$ and $b$, shows synapses where presynaptic profiles are filled with small vesicles and make contact on gly- 

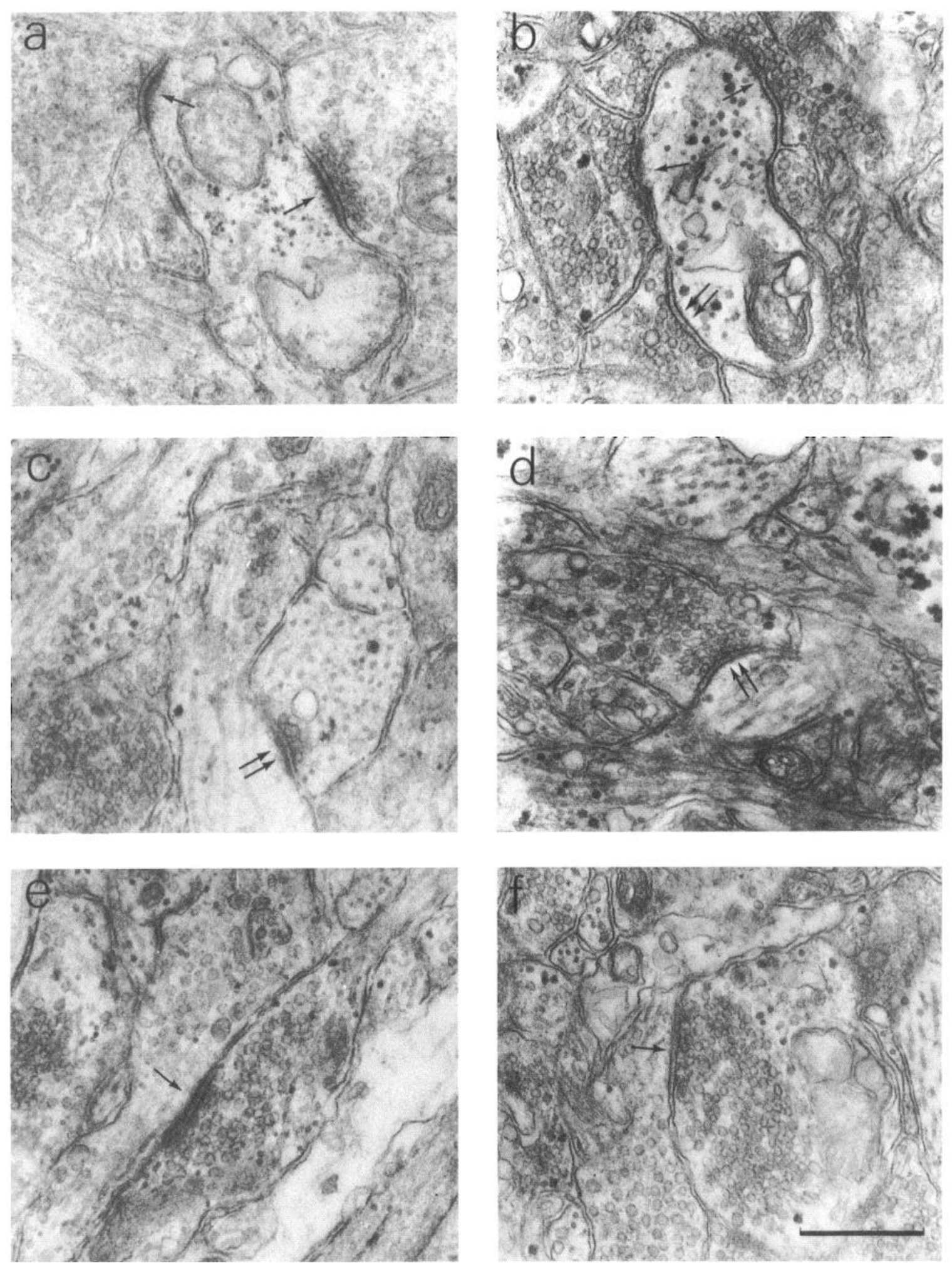

\section{NMDA}

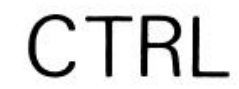

Figure 3. Examples of the range of synaptic types affected by NMDA treatment. $a, c$, and $e$ are high-power views of synapses in NMDA-treated, doubly innervated tecta. $b, d$, and $f$ are similar fields in sham-treated, doubly innervated tecta. $a$ and $b$ show synapses containing small vesicles making contact on glycogen-containing postsynaptic profiles (indicated by the single arrows). In both photographs the presynaptic profiles are filled with vesicles but the density of vesicle packing near the active zone is greater in $a$ than in $b . c, d$, and also the contact indicated by the double arrows in $b$ show synapses with small to large round vesicles and occasional dense-core vesicles. Notice again the tendency toward greater packing of vesicles near the presynaptic active zone in $c$ as compared to $b$ and $d$. $e$ and $f$ show synapses with small round vesicles making contact on postsynaptic profiles containing medium-sized round vesicles. All EM ultrathin sections photographed here were approximately $60 \mathrm{~nm}$ thick. Arrows point from postsynaptic to presynaptic profiles. Scale bar, $0.5 \mu \mathrm{m}$. 

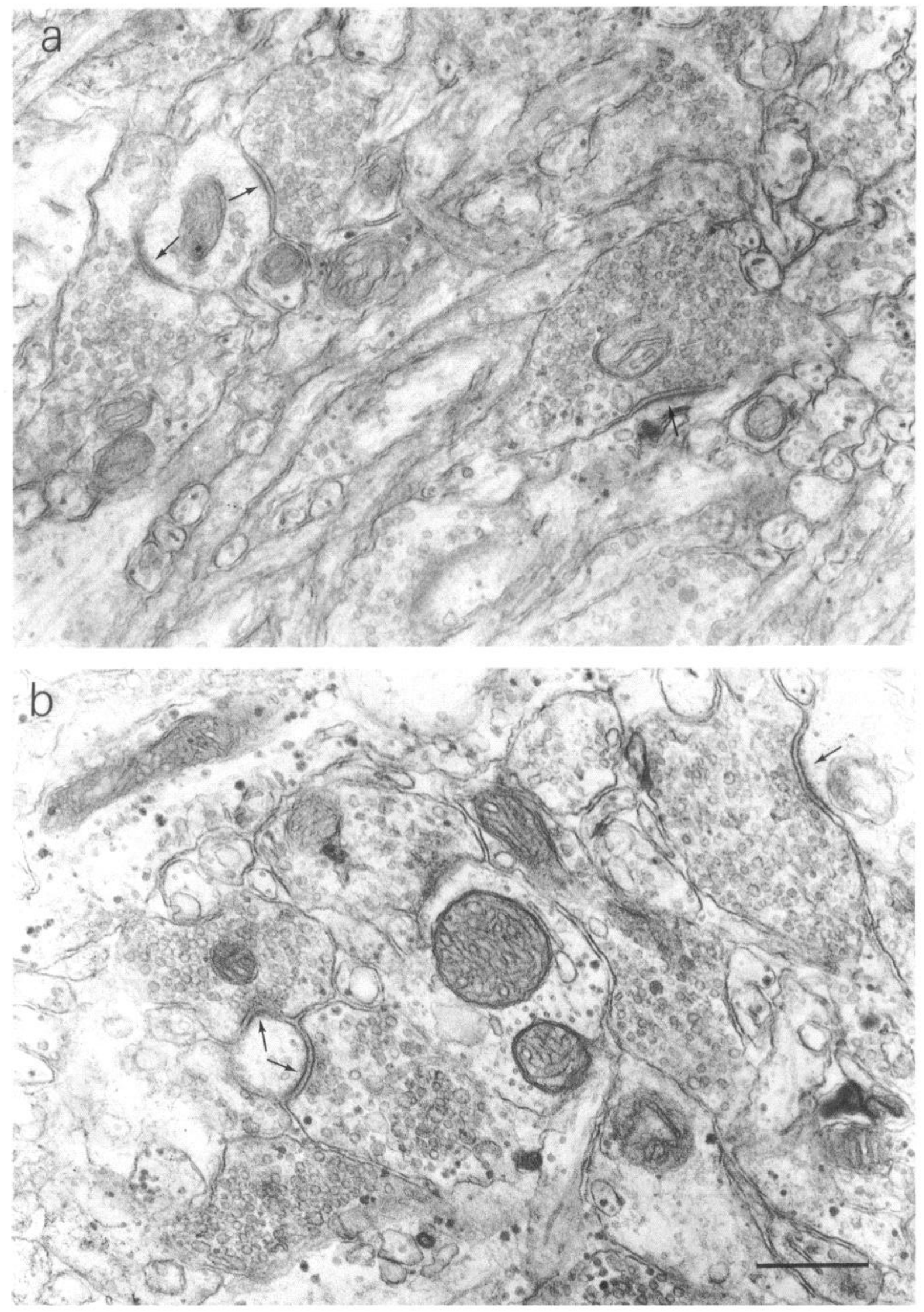

Figure 4. Neuropils unaffected by NMDA treatment. $a$, A field from the deeper layer 5 of an NMDA-treated doubly innervated tectum. $b$, A field from the superficial neuropil of an NMDA-treated "singly innervated" tectum of a two-eyed animal. Arrows point from postsynaptic to presynaptic profiles. Scale bar, $0.5 \mu \mathrm{m}$. 


\begin{tabular}{|c|c|c|c|}
\hline & 1 on 1 & 2 on 1 & $\geq 3$ on 1 \\
\hline \multicolumn{4}{|c|}{ Total number of cases } \\
\hline NMDA-1 & 468 & 75 & 5 \\
\hline NMDA-2 & 621 & 90 & 9 \\
\hline NMDA-3 & 663 & 96 & 11 \\
\hline CTRL-1 & 589 & 52 & 2 \\
\hline CTRL-2 & 522 & 54 & 8 \\
\hline CTRL-3 & 819 & 92 & 7 \\
\hline \multicolumn{4}{|c|}{ Percentage of total synapses } \\
\hline NMDA-1 & $73.82 \%$ & $23.66 \%$ & $2.52 \%$ \\
\hline NMDA-2 & $74.82 \%$ & $21.69 \%$ & $3.49 \%$ \\
\hline NMDA-3 & $74.41 \%$ & $21.55 \%$ & $4.04 \%$ \\
\hline Mean & $74.35 \%$ & $22.30 \%$ & $3.35 \%$ \\
\hline SD & $0.50 \%$ & $1.18 \%$ & $0.77 \%$ \\
\hline CTRL-1 & $84.14 \%$ & $14.86 \%$ & $1.00 \%$ \\
\hline CTRL-2 & $79.69 \%$ & $16.49 \%$ & $3.82 \%$ \\
\hline CTRL-3 & $79.90 \%$ & $17.95 \%$ & $2.15 \%$ \\
\hline Mean & $81.25 \%$ & $16.43 \%$ & $2.32 \%$ \\
\hline SD & $2.51 \%$ & $1.55 \%$ & $1.42 \%$ \\
\hline
\end{tabular}

cogen-containing postsynaptic profiles, $c$ and $d$ show synapses with small to large round vesicles with occasional dense-core vesicles (also see the synapse labeled with double arrows in Fig. $3 b$ ), and $e$ and $f$ show synapses with small round vesicles making contact on postsynaptic profiles containing medium round vesicles. These synaptic types are often seen in the neuropils. The heterogeneity of these synapses suggests that the thickening of synaptic densities is not restricted to only a subset of the synaptic types identified morphologically in the superficial neuropil of normal frogs (Hughes, 1990). The difference in pre- and postsynaptic densities was apparent in synapses within the superficial neuropil of the NMDA-treated tecta irrespective of whether they were located deep within neuropil layer 7 and 8 or in superficial regions of tectal layer 9 . However, this difference was not apparent in the deeper plexiform layer, layer 5 , where nonretinal inputs from tegmentum, contralateral tectum, and thalamus terminate (Fig. 4a) (Lázár, 1984). In other words, this pronounced change in synaptic morphology appeared to be restricted to the superficial neuropil of tecta where retinal projections terminate and where most of nonretinal converging inputs are indirectly driven by the eyes.

The data presented above indicated to us that the chronic application of NMDA in Elvax slices was altering the appearance of synapses in the superficial retinotectal neuropil in a manner that could not be accounted for merely by the continuous mechanical presence of the Elvax plastic. There remained the possibility, however, that the exogenous NMDA was altering the staining properties of the neuropil directly through an effect that was independent of the morphological pruning of axonal branches. To control for this possibility we performed EM surveys of the superficial neuropil in NMDA-treated, "singly innervated" tecta of two normal two-eyed animals. The earlier light microscope analyses of axonal arbor morphology in these singly innervated tecta found no evidence of branch pruning after chronic NMDA treatment (Cline and Constantine-Paton, 1990). As shown in Figure $4 b$, no signs of the pronounced pre-

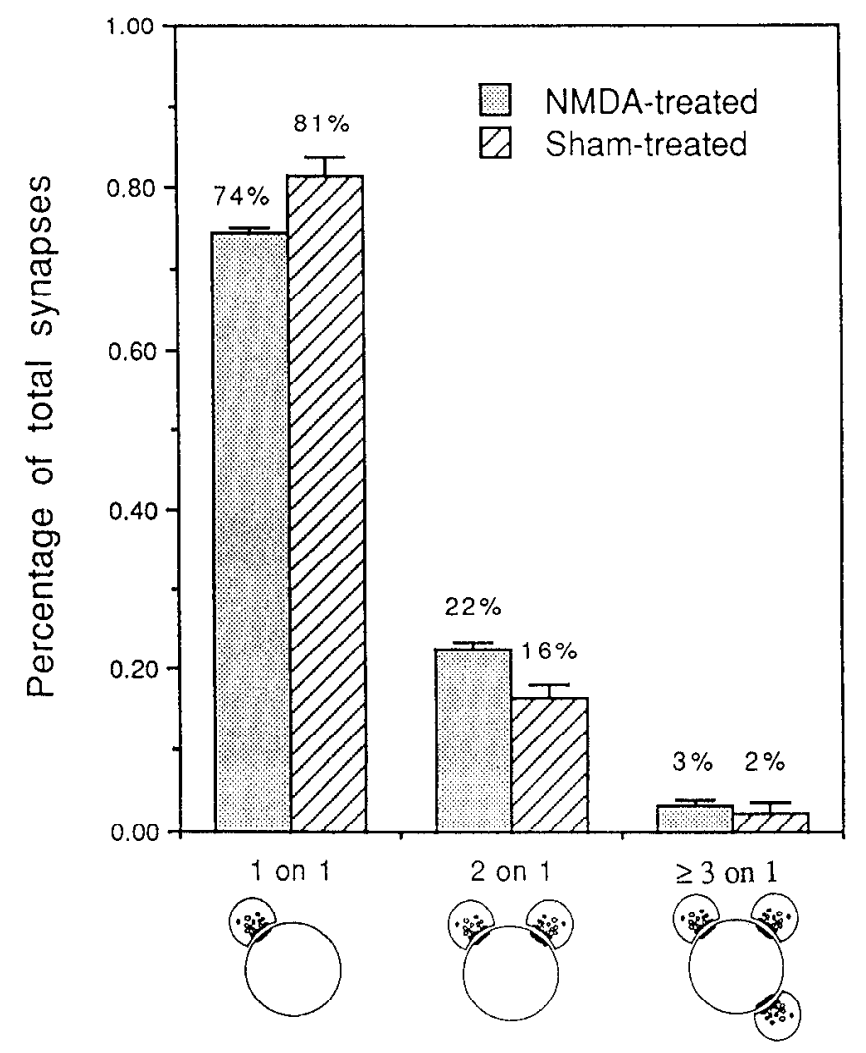

Figure 5. Chronic NMDA treatment effects on synaptic "clustering" in doubly innervated tecta. The data are shown as percentage of total synapses involved in each "clustering" category. NMDA treatment increases the number of presynaptic profiles observed in association with single postsynaptic profiles. The most conspicuous change is a shift from the 1 on 1 category to the 2 on 1 category. Error bars represent SD.

and postsynaptic densities observed in the doubly innervated tecta were seen in tecta of the two-eyed animals chronically treated with NMDA. The fine structure of synapses in these tecta appeared normal, suggesting that the effects of exogenous NMDA are associated only with the pruning of retinal axonal arbors.

Figure 3, $a$ and $c$, also illustrates a more subtle morphological change that was seen in many of the synapses in NMDA-treated, doubly innervated tecta. In these tecta, profiles with synaptic vesicles aggregated densely immediately adjacent to the presynaptic sites were more often seen. Unfortunately, this is only a suggestion from our observations. We were unable to obtain quantitative data indicating the magnitude of the NMDA treatment effects on synaptic vesicles, because thickened presynaptic densities often made it difficult to measure the number of vesicles and their distance from the synaptic release sites.

Initially, low-power electron micrographs of NMDA-treated and control neuropils in doubly innervated tecta gave the impression that the experimental tecta contained a larger than normal number of synaptic active zones clustered on the same postsynaptic profile. In order to determine if this impression reflected a significant alteration of synaptic complexes, a detailed stereological sampling of the three control and the three NMDAtreated doubly innervated neuropils was undertaken. The superficial neuropil of the tecta was sampled as described in Materials and Methods.

We scored each sample field for "synaptic clustering" by counting the number of presynaptic profiles converging on the 
(a)

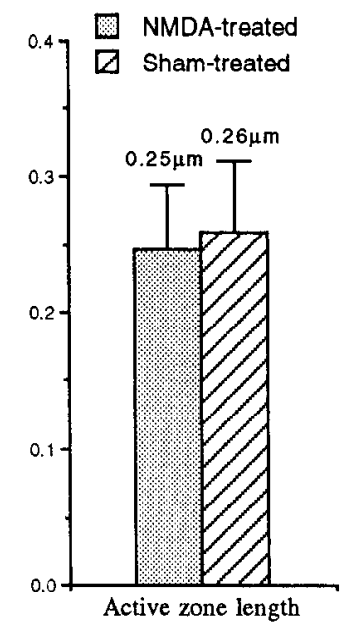

(b)

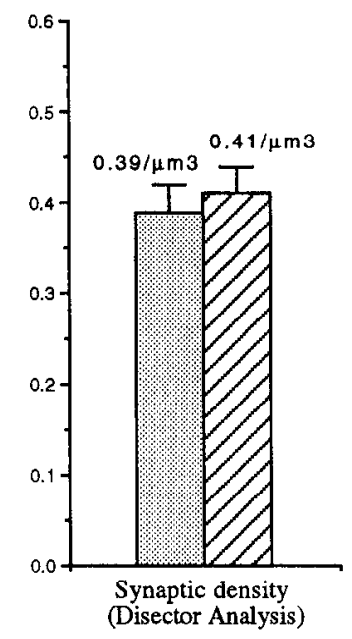

Figure 6. Lack of chronic NMDA treatment effects on active zone size or overall synaptic density. $a$, The length of synaptic active zones. $b$, Synaptic density estimated by disector analysis. Both parameters were statistically unchanged in NMDA-treated versus sham-treated striped tecta. The unit for synaptic density is the number of synapses per $\mu \mathrm{m}^{3}$, and the unit for the length of synaptic active zones is micrometers. Error bars represent $\mathrm{SD}$.

same postsynaptic profile (see the illustration of Fig. 5). Thus "1 on I" identified a single presynaptic profile on a postsynaptic profile, whereas " 2 on 1" refers to two presynaptic profiles converging on the same postsynaptic profile, and so on. The results of this analysis are shown in Table 1 as the total number of cases for each "clustering" category, and as the percentage of total synapses involved in each category. Figure 5 summarized the results. The data reveal that the number of presynaptic profiles observed in association with single postsynaptic profiles was increased in the NMDA-treated tecta. This change was highly significant ( $\chi^{2}$ test, $p<0.005$ ). As can be seen in Figure 5 , the most conspicuous shift occurred between the 1 on 1 category to the 2 on 1 category.

There are two quantitative alterations of synapses between control and NMDA-treated doubly innervated tecta that could produce the observed shift in clustering without actually changing the arrangement of the synapses in the NMDA-treated, doubly innervated tecta. First, if each synaptic active zone became larger with NMDA treatment, then the probability that a single ultrathin section would cut through more than one of the synaptic contacts converging on a single postsynaptic process would increase. This would produce an increased incidence of 2 on 1 or greater, but there would be no real change in the arrangement of the synapses. To examine this possibility, we measured the

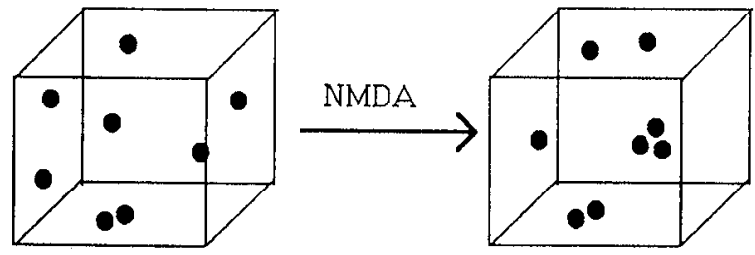

Figure 7. Diagrammatic illustration of the changes in synaptic arrangements that occur in NMDA-treated doubly innervated neuropils. Each $d o t$ in the cubic volumes of neuropil represents a synaptic active zone. Chronic NMDA treatment does not change the size or density of synaptic active zones; however, it increases synaptic clustering.

mean length of all synaptic active zones appearing in the sample photomicrographs from cach animal. As shown in Table 2 and Figure $6 a$, the size of synaptic active zone was unchanged in NMDA versus control tecta (ANOVA test, $0.1<p<0.25$ ). Second, an increase in the density of synapses in NMDA compared to control tecta could also produce the observed increase in clustered synaptic profiles without actually changing the synaptic arrangement. We tested this possibility by estimating synaptic density in the control and NMDA-treated neuropils using the disector analysis. The results are shown in Table 3 and Figure $6 b$. Animals with NMDA treatment have a mean synaptic density value of $0.39 \pm 0.03$ per $\mu \mathrm{m}^{3}$, which is also not significantly different from the control of $0.41 \pm 0.03$ per $\mu \mathrm{m}^{3}$ (Mann-Whitney $U$ test, $p=0.275$ ).

Figure 7 shows a diagram that depicts the implication of these quantitative analyses. In doubly innervated tecta, chronic NMDA treatment does not change the size or the overall density of synaptic active zones; however, it does significantly increase the incidence with which they cluster together in the tecta.

It would be satisfying to label completely all retinal terminals in these animals with HRP in order to obtain a specific figure indicating how many of the affected inputs are retinal in origin, or how frequently retinal inputs participate in the synaptic clusters. However, reliable HRP filling of all retinal terminals is impossible in these amphibian animals even when the retinal projection is from the normal eye. Moreover, filling of both retinal projections with HRP in the doubly innervated tectum would make it very difficult to verify that the tectum was indeed doubly innervated. In addition, this also makes it impossible to reconstruct the morphology of single RGC axonal arbors to show the branch pruning effects of NMDA treatment.

We could assure in this study, however, that all the tecta examined had a few type IV RGCs filled with HRP from the supernumerary retina. Many of these labeled terminals contributed to both the qualitative and quantitative observations we report above. Thus, we can say that this type of retinal terminal participates in synapses that show thickened pre- and postsyn-

Table 2. Mean length of synaptic active zones

\begin{tabular}{lcccccc} 
& \multicolumn{1}{c}{ Animal } \\
\cline { 2 - 7 } & NMDA-1 & NMDA-2 & NMDA-3 & CTRL-1 & CTRL-2 & CTRL-3 \\
\hline Total area sampled $\left(\mu \mathrm{m}^{2}\right)$ & 6709 & 7350 & 7350 & 6770 & 5835 & 10570 \\
Total synapses analyzed & 686 & 898 & 945 & 748 & 690 & 1092 \\
Mean length $(\mu \mathrm{m})$ & 0.24 & 0.25 & 0.25 & 0.25 & 0.26 & 0.27 \\
SD & 0.08 & 0.09 & 0.08 & 0.08 & 0.09 & 0.10 \\
\hline
\end{tabular}

ANOVA: $F(1,4)=F$ value for treatment $=5.23 ; F(1,4)$ at $0.05=7.71 ; 0.1<p<0.25$. 
Table 3. Number of synapses per unit volume

\begin{tabular}{lllllll} 
& \multicolumn{1}{l}{ Animal } & & & \\
\cline { 2 - 7 } & NMDA-1 & NMDA-2 & NMDA-3 & CTRL-1 & CTRL-2 & CTRL-3 \\
\hline Number of chosen areas & 112 & 128 & 122 & 118 & 114 & 118 \\
$\Sigma$ TOPs & 151 & 195 & 223 & 163 & 138 & 162 \\
$\Sigma$ Disector volumes $\left(\mu \mathrm{m}^{3}\right)$ & 411.0 & 514.9 & 535.1 & 414.7 & 311.7 & 396.0 \\
Synaptic density $\left(\# / \mu \mathrm{m}^{3}\right)$ & 0.37 & 0.38 & 0.42 & 0.39 & 0.44 & 0.41 \\
\hline
\end{tabular}

Mann-Whitney $U$ test, $p=0.275$.

aptic densities. We also observed these labeled terminals participating in synapse clusters where at least another input was converging on the same postsynaptic process (Fig. 8). Thus, at least some of the terminals in these clusters are retinal in origin and, as we discuss in more detail below, NMDA receptors are probably involved in mediating both retinal and nonretinal synaptic transmission. Thus, the actual proportion of retinal versus nonretinal inputs affected by our treatment may not be a major factor in determining the conclusions that can be drawn from these results.

\section{Discussion}

The EM analyses presented in this report document qualitative changes in the appearance of synapses within developing doubly innervated tecta when they are treated chronically with low doses of the agonist for the NMDA receptor. These effects are absent from the deep neuropil of the same tecta where nonretinal inputs terminate, and cannot, therefore, represent some generalized response of the tectal lobe to the treatment. It is also important to note that the changes are not a simple response to exogenous NMDA because the effects are absent from similarly treated superficial tectal neuropils if the tecta are innervated by only one eye. The qualitative changes in pre- and postsynaptic densities are only apparent in doubly innervated neuropils, where considerable pruning of synapse-bearing branches of retinal axonal arbors is observed at the light microscope level (Cline and Constantine-Paton, 1990). We found no signs of increases in degenerative responses such as vacuolated nuclei, phagocytotic processes, or electron-dense profiles (Peters et al., 1991) in the
NMDA-treated tecta. In short, our data rule out the possibility that NMDA is having a toxic effect on the developing retinotectal neuropil or that the exogenous agonist is acting directly on the retinal axons or the tectal dendrites themselves. Rather, the present observations reinforce the earlier light microscope study suggesting that structural changes are likely to be mediated through the postsynaptic cells: the changes only occur in striped tecta where activity correlations among converging inputs are low to begin with (Cline and Constantine-Paton, 1990). Synaptic fine structure is altered only in neuropil where axonal terminal arbors have been "pruned."

A number of factors have to be taken into consideration in order to interpret the present observations. For example, most of the excitatory drive to the superficial layers of the amphibian optic tectum comes from the RGC terminals (Grüsser and Grüsser-Cornehls, 1976), whose contacts make up roughly $60 \%$ of the synapses present in the neuropil (Ostberg and Norden, 1979). Thus, any alteration in the synaptic contact area of the RGCs within the tectum could significantly affect the level of tectal cell activation. In fact, light microscope reconstructions and EM analyses of single RGC axonal terminals from doubly innervated tecta have shown that within each arbor the distal branches contain most of the synaptic contacts. Pruning of the distal branches observed at the light microscopic level after NMDA treatment results in a significant loss in the total synaptic contact area of individual RGC axonal arbors when surveyed at the EM level (Yen and Constantine-Paton, 1991; L.-H. Yen and Constantine-Paton, unpublished observations).

The observed reduction in axonal terminal branching and
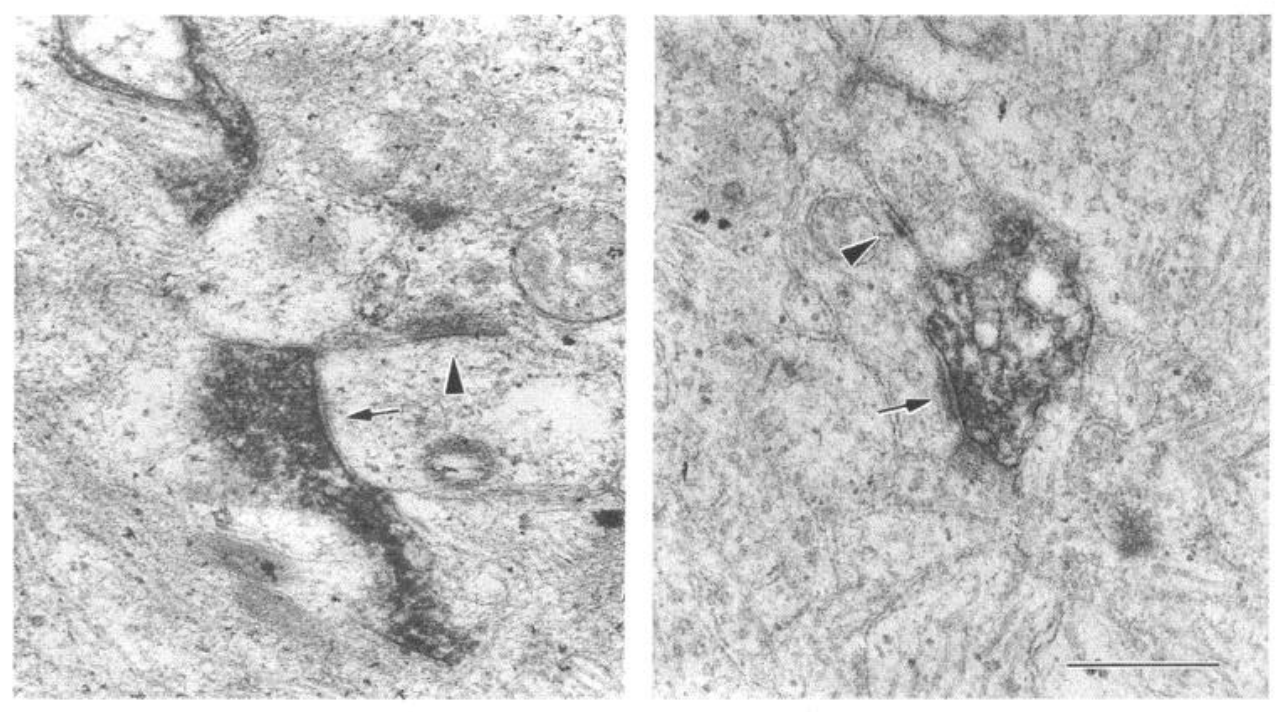

Figure 8. HRP-filled retinal axons each making a synapse (arrow) on a postsynaptic profile in layer 8 of an NMDA-treated, doubly innervated tectum. In each photograph, the same postsynaptic profile receives another synapse (arrowhead) from a different axon. Scale bar, $0.5 \mu \mathrm{m}$. 
therefore in the total synaptic contact area of a neuron's axon, however, has a number of implications for the function of the synapses that are maintained by thosc same axons. Several lines of evidence indicate that, as a result of such reductions, each of the remaining synaptic release sites may be proportionately more "effective" than their counterparts in extensively arborized axon terminals. For example, the molecules and organelles necessary for synaptic function are likely to be transported down the major axon cylinder in quantities that are relatively independent of the axoplasmic volume available (Weiss and Hiscoe, 1948). Consequently, each synapse-bearing branch in a poorly arborized axonal terminal, as opposed to a richly branched axonal terminal, should have more essential cytoplasmic constituents per synaptic release site. In addition, failure of action potential spike conduction is predicted to occur at axonal branch points because of the decreased current density in these regions (Khodorov and Timin, 1975; Parnas and Segev, 1979). Physiological investigations have provided experimental support for this phenomenon (Parnas, 1972; Grossman et al., 1973, 1979; Dyball et al., 1987; Stoney, 1990). Therefore, assuming all other passive cable properties of the axon remain constant, spike invasion to all synaptic release sites is likely to occur with higher probability in a less arborized as compared to a richly arborized terminal.

Several studies have indicated that pruning of axonal terminals is associated with an increase in the efficacy of the surviving terminals. In chick, the developmental pruning of multiple inputs of the cochlea nerve axons onto the neurons of the nucleus magnocellularis is associated with a faster rise time and a bigger EPSP from the remaining input. Although in this case the pruning of the afferents is associated with synapses that are larger (Jackson and Parks, 1982). At mouse and frog neuromuscular junction, pruning of motor axons is associated with an increase in the efficacy of the surviving nerve terminals (Herrera and Grinnell, 1980, 1981; Pockett and Slack, 1982). An inversc rclationship between the extent of arborization and the efficacy of each synaptic contact is also consistent with findings suggesting that synaptic junctions formed by sprouted motor axons are relatively weak (Bennett and Raftos, 1977). It is interesting that these "sprouted" motor axons appear to be easily displaced by regenerating nerves that are maintaining less arborization (Wigston, 1980; Haimann et al., 1981). Thus, richly arborized arbors may be less effective than "pruned" arbors at stabilizing individual synapses on a contested target. This is consistent with observations in the hamster showing that RGC axons that have had their terminal arbor space in the superior colliculus "pruned" by tectal ablation are extremely effective at sprouting new synapse bearing branches in the diencephalon (Sabel and Schneider, 1988).

These observations by other investigators suggest that, because of the pruning of RGC arbors in doubly innervated tecta treated with $\mathrm{NMD \Lambda}$, each of the remaining RGC synapses is likely to be more effective. They are likely to have more frequent action potential invasion than their counterparts in unpruned arbors, and they are likely to release more transmitter in response to each spike. In other words, despite the downregulation of NMDA receptor effectiveness that occurs with the NMDA chronic treatment (Debski et al., 1991; Hickmott and Constantine-Paton, 1991), the synapses remaining after pruning may be equally or perhaps even more efficient at driving NMDA receptors than their unpruned counterparts in either sham-treated doubly innervated tecta or singly innervated tecta treated with NMDA.
We believe that this increased "use" and/or "efficacy" of individual synapses may partially account for the qualitative changes in pre- and postsynaptic densities seen in the present study. A number of investigations have reported correlations between neural activity levels and morphological characteristics of synapses similar to those we report here. For example, in the visual cortex, the postnatal elaboration of the presynaptic grid has been found to be halted or stalled by dark rearing (Muller et al., 1981), and the morphological maturation of RGC synapses in the kitten dorsal lateral geniculate nucleus is similarly retarded when RGC activity is blocked with intraocular injections of TTX (Kalil et al., 1986). In addition, visual learning that should be associated with increased synaptic use has been shown to be associated with increases in the thickness of the postsynaptic density in the visual cortex (Vrensen and Cardozo, 1981).

Increases in the "use" or the "efficacy" of synapses have also been positively correlated with an increase in synaptic vesicle packing near the presynaptic membrane. Thus, the number of synaptic vesicles per synapse increases with the acquisition of new song behaviors in the nucleus robustus archistriatalis of forebrain, the area known to be involved in song control in canaries (DeVoogd et al., 1985). In the anteroventral cochlear nucleus of rodent the density of the presynaptic vesicle fusion sites per active zone as assayed by freeze-fracture method increases with increasing levels of acoustic stimulation (Gully, 1978). Bailey and Chen $(1983,1988)$ have shown that long-term sensitization of the sensory inputs onto Aplysia gill withdrawal motor neurons increases the number of synaptic vesicles closely adjacent to the synaptic active zone, while long-term and shortterm habituation is associated with a depletion of synaptic vesicles immediately adjacent to the active zones. In addition, one study that correlated physiological and fine-structural changes in synapse development at identified neuromuscular junctions in culture noted that increased synaptic efficacy is associated with more vesicle clustering at release sites and increases in the thickness of postsynaptic density (Buchanan et al., 1989).

Two observations in the present study, however, cannot be accounted for by simply postulating increased "use" and/or "efficacy" of the synapses that remain within pruned arbors arising from the retina. One is the finding that the majority of synaptic active zones in the affected tcctal ncuropils show the increases in pre- and postsynaptic densities despite the fact that at least some of these synapses probably come from inputs other than the RGCs themselves. If our reasoning concerning the cause of the increased pre- and postsynaptic thickening is correct, then the widespread occurrence of such thickening implies that nonretinal as well as retinal afferents have been pruned in doubly innervated tecta treated with NMDA. Currently, data are not available to address this possibility since the terminal arbors of nonretinal inputs have not been reconstructed at the light microscopic level. Nevertheless, the explanation for pruning of retinal terminals, that is, decreased ability of tectal neurons to stabilize inputs with low activity correlations, could account equally well for pruning of nonretinal inputs. This is because the work of others in mature animals (Rubinson, 1968; Wilczynski and Northcutt, 1977) and our own work in tadpoles and young frogs (Debski and Constantine-Paton, 1993) indicate that most of the nonretinal afferents to the tectum arise from diencephalic and midbrain regions that are themselves targets of tectal efferents. In addition, recent whole-cell patch-clamping studies have demonstrated that an NMDA receptor-mediated 
component contributes to both the monosynaptic and polysynaptic excitatory responses of tectal neurons driven by optic tract stimulation (Hickmott and Constantine-Paton, 1993). Thus, the situation that causes poorly correlated retinal inputs to be pruned might be expected to also cause many of the nonretinal inputs that are polysynaptically driven by that same visual input to be pruned as well. Moreover, these inputs do not have to be glutamatergic to be affectcd by changes in NMDA receptor function. Work on the cholinergic isthmiotectal projection of the frog Xenopus has demonstrated that disruption of NMDA receptor function can also disorganize the normal development of this convergent projection (Udin and Scherer, 1990). In short, our results appear to support the idea that the majority of inputs to the superficial neuropil of doubly innervated tecta, even ones that do not arise from the retina and some that may not use glutamate as the transmitter, may require activation of NMDA receptors on tectal neurons in order to become stabilized. These inputs, like the retinal afferents, are probably similarly pruned under conditions where NMDA receptor effectiveness is low.

The second set of data in the present study that cannot be easily explained as a result of RGC terminal arbor pruning is the increase in the incidence of two or more presynaptic processes converging on the same postsynaptic target. We think that this obscrvation may be an additional evidence in support of the idea that the population of synapses surviving in doubly innervated tecta chronically treated with NMDA is enriched for contacts that share the same tectal target along with highly correlated neighbors. Our reasoning is as follows. Data from many different kinds of experiments indicate that the mechanism that stabilizes young synapses on the basis of their ability to induce significant depolarizations in the target cell is competitive; that is, an input that is not active when the target cell is depolarized is actually less likely to survive on that target (Wiesel and Hubel, 1965; Stent, 1973; Lo and Poo, 1991; Dan and Poo, 1992). There is also evidence that, if the target cell cannot be activated by an input, then activation of that input actually results in a decreased effectiveness of the active over the inactive contact (Reiter and Stryker, 1988). When two or more excitatory inputs converge on the same postsynaptic process within a distance of $1 \mu \mathrm{m}$ of onc another, it seems very likely that they will both be affected by the depolarization produced by either one of them in that local region of dendrite. Consequently, unless synaptogenesis in the optic tectum occurs without synaptic competition, which is extremely unlikely (Prestige and Willshaw, 1975; Udin and Fawcett, 1988), one would not expect sets of uncorrelated or poorly correlated inputs to be clustered. We cannot prove that these synaptic clusters have unusually highly correlated activity among the participating synapses, because the identity and the activity patterns of the inputs are not known. Nevertheless, we know that some of the inputs contributing to these synaptic clusters are indeed retinal, because they are labeled with HRP from the retina. Furthermore, available information on retinotopic mapping in normal and striped tecta (Law and Constantine-Paton, 1981) and on the reciprocal connections in which tectal cells participate indicate that highly correlated inputs are likely to be present in local tectal regions. We suggest, therefore, that the increased incidence of two or more presynaptic inputs on the same postsynaptic target, in a situation where the size of synaptic active zone or overall synaptic density does not change, does in fact represent an increased incidence of highly correlated inputs. This is the result expected if the rela- tively poorly correlated inputs were selectively eliminated in the superficial neuropil as a result of the chronic NMDA treatment.

In summary, our data demonstrate that increased pre- and postsynaptic thickening and increased synaptic clustering accompany the branch loss of retinal axonal arbors following chronic NMDA treatment of tecta innervated by two retinas. We believe that the most reasonable explanation for these data is that the NMDA treatment enriches the neuropil for wellcorrelated inputs by decreasing the effectiveness of NMDA receptors on tectal neurons. Thus, synapses that have relatively lower activity correlations with their neighbors cannot be stabilized and are eventually eliminated. An alternative explanation for these data is that the observed changes are a simple response to exogenous NMDA by the tectal neuropil without involving this activity-dependent mechanism. However, this is unlikely because the observed effects are absent from similarly treated tectal neuropils innervated by only one eye. These tecta innervated by a single retina do not have the reduced correlated activity among converging inputs as induced by double innervation.

\section{References}

Bailey $\mathrm{CH}$, Chen M (1983) Morphological basis of long-term habituation and sensitization in Aplysia. Science 220:91-93.

Bailey CH, Chen M (1988) Morphological basis of short-term habituation in Aplysia. J Neurosci 8:2452-2459.

Bear M, Kleinschmidt A, Gu Q, Singer W (1990) Disruption of experience-dependent modifications in striate cortex by infusion of an NMDA receptor antagonist. J Neurosci 10:909-924.

Bennett M, Raftos J (1977) The formation and regression of synapses during the reinnervation of axolotl striated muscle. J Physiol (Lond) 265:261-295.

Buchanan J, Sun Y-A, Poo M-M (1989) Studies of nerve-muscle interactions in Xenopus cell culture: fine structure of early functional contacts. J Neurosci 9:1540-1544.

Changeux J-P, Danchin A (1976) Selective stabilization of developing synapses as a mechanism for the specification of neural networks. Nature 264:705-712.

Cline HT, Constantine-Paton M (1989) NMDA receptor antagonists disrupt the retinotectal topographic map. Neuron 3:413-426.

Cline HT, Constantine-Paton M (1990) NMDA receptor drug treatment alters RGC terminal morphology in vivo. I Neurosci 10:11971216.

Cline HT, Debski EA, Constantine-Paton M (1987) NMDA receptor antagonist desegregates eye-specific stripes. Proc Natl Acad Sci USA $84: 4342-4345$.

Coggeshall R (1992) A consideration of neural counting methods. Trends Neurosci 15:9-13.

Constantine-Paton M, Ferrari-Eastman P (1987) Pre- and postsynaptic correlates of interocular competition and segregation in the frog. J Comp Neurol 255:178-195.

Dan Y, Poo M-m (1992) Hebbian depression of isolated neuromuscular synapses in vitro. Science 256:1570-1573.

Debski EA, Constantine-Paton M (1993) The development of nonretinal afferent projections to the frog optic tectum and the substance P immunoreactivity of tectal connections. Dev Brain Res 72:21-39.

Debski EA, Cline HT, McDonald JW, Constantine-Paton M (1991) Chronic application of NMDA decreases the NMDA sensitivity of the evoked potential in the frog. J Neurosci 11:2947-2957.

DeVoogd TJ, Nixdorf B, Nottebohm F (1985) Synaptogenesis and changes in synaptic morphology related to acquisition of a new behavior. Brain Res 329:304-308.

Dyball R, Grossmann R, Leng G, Shibuki K (1987) Action potential conduction failure in the rat neurohypophysis. J Physiol (Lond) 388: $13 \mathrm{p}$.

Grossman Y, Spira M, Parnas I (1973) Differential flow of information into branches of a single axon. Brain Res 64:379-386.

Grossman Y, Parnas I, Spira M (1979) Differential conduction block in branches of a bifurcation axon. J Physiol (Lond) 295:283-305. 
Grïsser O-J, Grüsser-Cornehls U (1976) Neurophysiology of the anuran visual system. In: Frog neurobiology, pp 297-368. New York: Springer.

Gully R (1978) Changes in the presynaptic membrane of the synapses of the anteroventral cochlear nucleus with different levels of acoustic stimulation. Brain Res 146:373-379.

Gundersen HJG et al. (1988) The new stereological tools: disector, fractionator, nucleator and point sampled intercepts and their use in pathological research and diagnosis. Acta Pathol Microbiol Immunol Scand 96:857-881.

Hahm J-O, Langdon RB, Sur M (1991) Disruption of retinogeniculate afferent segregation by antagonists to NMDA receptors. Nature 351 : $568-570$.

Haimann C, Mallart A, Ferre J, Zilber-Gachelin N (1981) Interaction between motor axons from two different nerves reinnervating the pectoral muscle of Xenopus laevis. J Physiol (Lond) 310:257-272.

Herrera A, Grinnell A (1980) Transmitter release from frog motor nerve terminals depends on motor unit size. Nature 287:649-651.

Herrera A, Grinnell A (1981) Contralateral denervation causes enhanced transmitter release from frog motor nerve terminals. Nature 291:495-497.

Hickmott P, Constantine-Paton M (1991) Quantitative analysis of agonist-evoked currents in identified tectal neurons of Rana pipiens. Soc Neurosci Abstr 17:1134.

Hickmott P, Constantine-Paton M (1993) The contributions of NMDA, non-NMDA, and GABA receptors to postsynaptic responses in neurons of the optic tectum. J Neurosci, in press.

Hughes T (1990) A light- and electron-microscopic investigation of the optic tectum of the frog, Rana pipiens. I. The retinal axons. Vis Neurosci 4:499-518.

Jackson H, Parks T (1982) Functional synapse elimination in the devcloping avian cochlear nucleus with simultaneous reduction in cochlear nerve axon branching. J Neurosci 2:1736-1743.

Kalil RE, Dubin MW, Grayson S, Stark LA (1986) Elimination of action potentials blocks the structural development of retinogeniculate synapses. Nature 323:156-158.

Khodorov B, Timin E (1975) Nerve impulse propagation along nonuniform fibres. Prog Biophys Mol Biol 30:145-184.

Kleinschmidt A, Bear MF, Singer W (1987) Blockade of NMDA receptors disrupts experience-dependent plasticity of kitten striate cortex. Science 238:355-358.

Law MI, Constantine-Paton M (1981) Anatomy and physiology of experimentally produced striped tecta. J Neurosci 1:741-759.

Lázár G (1984) Structure and connections of the frog optic tectum. In: Comparative neurology of the optic tectum, pp 185-208. New York: Plcnum.

Lo Y-J, Poo M-m (1991) Activity-dependent synaptic competition in vitro: heterosynaptic suppression of developing synapses. Science 254 : 1019-1022.

Muller L, Pattislanno A, Vrensen G (1981) The postnatal development of the presynaptic grid in the visual cortex of rabbits and the effect of dark-rearing. Brain Res 205:39-48.

Ostberg A, Norden JJ (1979) Ultrastructural study of degeneration and regeneration in the amphibian tectum. Brain Res 168:441-455.

Parnas I (1972) Differential block at high frequency of branches of a single axon innervating two muscles. J Neurophysiol 35:903-914.

Parnas I, Segev I (1979) A mathematical model for conduction of action potentials along bifurcating axons. J Physiol (Lond) 295:323343.

Peachey LD (1958) Thin sections: I. A study of scction thickness and physical disortion produced during microtomy. J Biophys Biochem Cytol 4:233-242.
Peters A, Palay SL, Webster HD (1991) The fine structure of the nervous system, 3d ed, p 367. London: Oxford UP.

Pockett S, Slack J (1982) Pruning of axonal trees results in increased efficacy of surviving nerve terminals. Brain Res 243:350-353.

Prestige M, Willshaw D (1975) On a role for competition in the formation of patterned neural connexions. Proc R Soc Lond [Biol] 190: 77-98.

Reh TA, Constantine-Paton M (1985) Eye-specific segregation requires neural activity in three-eyed Rana pipiens. J Neurosci 5:11321443.

Reiter RO, Styker MP (1988) Neural plasticity without postsynaptic action potentials: less active inputs become dominant when kitten visual cortex cells are pharmacologically inhibited. Proc Natl Acad Sci USA 85:3623-3627.

Rubinson K (1968) Projections of the optic tectum of the frog. Brain Behav Evol 1:529-561.

Sabel B, Schneider G (1988) The principle of "conservation of total axonal arborizations": massive compensatory sprouting in the hamster subcortical visual system after early tectal lesions. Exp Brain Res 73:505-518.

Shumway W (1940) Stages in the normal development of Rana pipiens. I. External forms. Anat Rec 83:39-315.

Silberstein G, Daniel C (1982) Elvax 40P implants: sustained, local release of bioactive molecules influencing mammary ductal development. Dev Biol 93:272-278.

Stent GS (1973) A physiological mechanism for Hebb's postulate of learning. Proc Natl Acad Sci USA 70:997-1001.

Sterio D (1984) The unbiased estimation of number and sizes of arbitrary particles using the disector. J Microsc 134:127-136.

Stirling R, Merrill E (1987) Functional morphology of frog retinal ganglion cells and their central projections: the dimming detectors. J Comp Neurol 258:477-495.

Stoney S (1990) Limitation on impulse conduction at the branch point of afferent axons in frog dorsal root ganglion. Exp Brain Res 80:512524.

Székely G, Lázár G (1976) Cellular and synaptic architecture of the optic tectum. In: Frog neurobiology. New York: Springer.

Taylor AC, Kollros JJ (1946) Stages in the normal development of Rana pipiens larvae. Anat Rec 94:7-23.

Udin SB, Fawcett JW (1988) Formation of topographic maps. Annu Rev Neurosci 11:289-327.

Udin SB, Scherer WJ (1990) Restoration of the plasticity of binocular maps by NMDA after the critical period in Xenopus. Science 249: 669-672.

Vrensen G, Cardozo JN (1981) Changes in size and shape of synaptic connections after visual training: an ultrastructural approach of synaptic plasticity. Brain Res 218:79-97.

Weiss P, Hiscoe (1948) Experiment on the mechanism of nerve growth. J Exp Zool 107:315-396.

Wiesel T, Hubel D (1965) Comparison of the effects of unilateral and bilateral eye closure on cortical unit responses in kittens. J Neurophysiol 28:1029-1040.

Wigston D (1980) Suppression of sprouted synapses in axolotl muscle by transplanted foreign nerves. J Physiol (Lond) 307:355-366.

Wilczynski W, Northcutt RG (1977) Afferents to the optic tectum of the leopard frog: an HRP study. J Comp Neurol 173:219-229.

Yen L-H, Constantine-Paton M (1991) EM analysis of single retinal ganglion cell terminals in NMDA-treated tecta Rana pipiens. Soc Neurosci Abstr 17:1134. 